\title{
Parasitological teeth for evolutionary problems
}

\section{Paul H. Harvey}

EvolutionARY biologists have much to gain from a closer association with parasitologists. It is not just that parasites have been overlooked for too long as a selective force, and that a considered assessment may show them to be at least partly responsible for a few of the problems that Darwin left us - sex, sexual selection and sociality have received attention recently. There is more to it than that. A parasitological perspective may shed light on evolutionary problems that do not necessarily involve one species parasitizing another. Graham Bell and Austin Burt's assessment of the evolutionary significance of $\mathrm{B}$ chromosomes, which was presented at a recent symposium on evolutionary approaches to parasitism*, nicely illustrates the point.

B chromosomes are distinguished from A chromosomes both by differing in number between individuals of the same species and by being unnecessary for the survival or reproduction of the individuals that possess them. They are extremely common, probably being present in $10-$ 15 per cent of eukaryotic species, and are typically small. Often they are extensively heterochromatized - pointing to genetic inertness. Even though they may have originated from the autosomes or sex chromosomes of the lineage in which they are found, B chromosomes have clearly evolved properties of their own. In several plant species, B chromosomes are lost from the root tissue but retained in the aerial parts. And, in the growing shoots, they may be lost from tissues that will become nonreproductive, yet increase in numbers in tissue that will give rise to the germ line. Similarly, whereas small univalent chromosomes and chromosome fragments tend to be lost during meiosis, $\mathrm{B}$ chromosomes may actually accumulate. In organisms ranging from grasshoppers to rye grass, the B chromosomes move preferentially during meiosis to those daughter nuclei that will give rise to germ cells rather than polar bodies or nongenerative nuclei.

Given this accumulation of B chromosomes in the germ cells, why do their numbers not increase from generation to generation? The answer seems to be that they reduce the darwinian fitness of their hosts. Cells with more DNA take longer to divide, and at least one study demonstrates that B chromatin adds about twice the amount of time to a typical cell division compared with a similar mass of $\mathrm{A}$

* British Society for Parasitology Autumn Symposium, City University, London, 29 September 1989. Organized by A. E. Keymer and A. F. Read chromatin. Morphological comparisons from a number of plant and animal species indicate that individuals with more $\mathrm{B}$ chromosomes usually exhibit delayed development, which is often accompanied by reduced vigour and fertility. In other words, B chromosomes bear all the hallmarks of successful parasites.

According to R. N. Jones and H. Rees (B-Chromosomes, Academic, London, 1982 ), the parasite theory for B chromosomes was suggested as long ago as 1945 by G. Östergren. Since then it has largely gone out of fashion and $\mathrm{B}$ chromosomes have been viewed as either of neutral selective value, or as being advantageous to the individuals or species that bear them. The parasite theory, however, does in fact seem successfully to accommodate recent observations. What is more, the theory makes fairly straightforward predictions that remain to be tested. For example, if B chromosomes are disadvantageous to their hosts, they should be lost from clonal lines and asexual species. In Bell and Burt's terminology, B chromosomes constitute not merely a disease, but a sexually transmitted disease.

The parasitological perspective on $\mathrm{B}$ chromosome evolution may lead to the reinterpretation of other observations. For example, there seems to be additive genetic variance for the presence and number of B chromosomes, which might be interpreted as differential resistance by particular sets of autosomes to invasion by B chromosomes. If that is so, the 'Red Queen' theory for the evolution of recombination (selection in response to everchanging environmental challenges) might be invoked: that is, we might expect individuals possessing $\mathrm{B}$ chromosomes to increase recombination among the autosomes, so that new genetic variants that are resistant to $\mathrm{B}$ chromosomes would be more likely to arise. At the same time, we should expect successful B chromosomes to reduce the rates of recombination in their hosts.

So far, the evidence bearing on these questions, as reviewed by Bell and Burt, shows no consistent patterns. Working out concise predictions, suitable comparative tests and experimental protocols are tasks for the future. But to ask whether the work will be carried out by parasitologists or evolutionary biologists depends on an outmoded distinction between scientists with identical perspectives.

Paul H. Harvey is in the Department of Zoology, University of Oxford, South Parks Road, Oxford OX1 3PS, UK.

\section{Earth, wind and fire}

Some time ago the 'hydrogen economy' was proposed as a way of avoiding pollution. Hydrogen would be made in bulk by the nuclear-powered electrolysis of water or, perhaps, by the chemical transformation of oil. A universal fuel, it would burn cleanly to harmless water.

This green dream soon faded into well deserved oblivion. Nuclear power has its own potential for environmental disaster, and the generation of hydrogen from oil liberates as much greenhouse-inducing carbon dioxide as simply burning the oil in the usual way. But Daedalus is now reviving the idea. His plan is to turn the oil into hydrogen while it is still in the ground.

The modern steam-reforming catalytic hydrogen process would be hard to employ on crude oil in situ. Steam, heat and catalyst would all have to be fed continuously down the borehole. But Daedalus recalls the old partial-oxidation process which burnt crude oil in a limited supply of pressurized oxygen with a little steam in it, giving mainly hydrogen and carbon dioxide. No catalyst or added heat was needed. So Daedalus plans to pump oxygen and water down a test oil well, ignite the mixture electrically at the bottom and collect the resulting hydrogen from a central exit pipe.

Deep in the Earth, the process will run under great hydrostatic pressure. Carbon dioxide and undesirable by-products will dissolve in excess condensed water, and the pressurized solution will soak back into the porous oil-bearing rock as fast as the oil flows out of it. So the nasties will stay underground; only hydrogen will be volatile enough to escape to the surface.

Geohydrogen would be tricky to distribute. Overland, it could travel through the pipelines that now take oil and gas. But as bulk sea-freight it would be troublesome. Daedalus proposes to tow it round the world in captive balloons.

A small tug could easily tow a big balloon full of hydrogen. Even better, the balloon might tow the tug. The wind direction often varies markedly with height, and by winching the balloon up to the right altitude, the tug could be pulled in any chosen direction - a wonderful advance on the traditional sailing ship. Once discharged at its destination, the deflated balloon could be stowed on board the tug for the journey back to the oil field.

As a major fuel, hydrogen could be piped around like domestic or industrial gas. It could provide heat, power and clean water simultaneously and economically, wherever they are needed, ideally by means of a fuel cell. All potential pollutants would be left behind in the oil fields. Future geologists, prospecting for what remains of the oil, may be puzzled to strike dirty soda water.

David Jones 